\title{
IL-32 $\gamma$ attenuates airway fibrosis by modulating the integrin-FAK signaling pathway in fibroblasts
}

\author{
Gyong Hwa Hong ${ }^{1,2+}$, So-Young Park ${ }^{3 \dagger}$, Hyouk-Soo Kwon², Bo-Ram Bang ${ }^{1,2}$, Jaechun Lee ${ }^{4}$, Sang-Yeob Kim ${ }^{1,5}$, \\ Chan-Gi Pack ${ }^{1,5}$, Soohyun Kim ${ }^{6}$, Keun-Ai Moon ${ }^{1,2}$, Tae-Bum Kim², Hee-Bom Moon ${ }^{2}$ and You Sook Cho ${ }^{2 *}$
}

\begin{abstract}
Background: Fibrosis in severe asthma often leads to irreversible organ dysfunction. However, the mechanism that regulates fibrosis remains poorly understood. Interleukin (IL)-32 plays a role in several chronic inflammatory diseases, including severe asthma. In this study, we investigated whether IL-32 is involved in fibrosis progression in the lungs.

Methods: Murine models of chronic airway inflammation induced by ovalbumin and Aspergillus melleus protease and bleomycin-induced pulmonary fibrosis were employed. We evaluated the degree of tissue fibrosis after

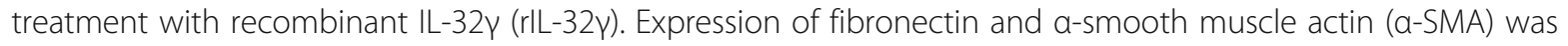
examined and the transforming growth factor (TGF)- $\beta$-related signaling pathways was evaluated in activated human lung fibroblasts (MRC-5 cells) treated with rlL-32y.

Results: $r$ IL-32 $\gamma$ significantly attenuated collagen deposition and a-SMA production in both mouse models. rIL-32 $\gamma$ inhibited the production of fibronectin and a-SMA in MRC-5 cells stimulated with TGF- $\beta$. Additionally, rlL-32 $\gamma$ suppressed activation of the integrin-FAK-paxillin signaling axis but had no effect on the Smad and non-Smad signaling pathways. rlL-32y localized outside of MRC-5 cells and inhibited the interaction between integrins and the extracellular matrix without directly binding to intracellular FAK and paxillin.
\end{abstract}

Conclusions: These results demonstrate that IL-32 $\gamma$ has anti-fibrotic effects and is a novel target for preventing fibrosis. Keywords: Interleukin-32y, Asthma, Airway inflammation, Subepithelial fibrosis, Pulmonary fibrosis

\section{Background}

Fibrosis, characterized by the accumulation of fibroblasts and excess extracellular matrix, is a common feature of various pathological states in many organs, resulting in dysfunction. Interstitial lung diseases and chronic inflammatory airway diseases of the lungs, such as severe asthma and chronic obstructive pulmonary disease (COPD), lead to sub-bronchial fibrosis and pulmonary fibrosis, both of which result in irreversible structural changes that affect patient survival [1-3]. Because lung fibrosis is mainly a consequence of chronic inflammation, therapeutic strategies have focused on preventing

\footnotetext{
* Correspondence: yscho@amc.seoul.kr

${ }^{\dagger}$ Gyong Hwa Hong and So-Young Park contributed equally to this work.

${ }^{2}$ Department of Internal Medicine, Division of Allergy and Clinical

Immunology, Asan Medical Center, University of Ulsan College of Medicine,

88 Olympic-ro 43-gil, Songpa-gu, Seoul 138-736, Korea

Full list of author information is available at the end of the article
}

inflammation by administering immunosuppressive agents or anti-inflammatory drugs, including corticosteroids $[4,5]$. However, recent studies have suggested that inflammation alone is not sufficient for inducing fibrosis development. Many studies showed that immunosuppressive therapies do not prevent lung fibrosis [6]. To date, targeting fibrosis itself has been unsuccessful.

Interleukin (IL)-32, initially described as NK4 generated by activated T cells or NK cells [7], is produced by various cells, including epithelial cells, endothelial cells, and macrophages. IL-32 induces the production of several pro-inflammatory mediators, such as tumor necrosis factor (TNF)- $\alpha$, IL- $1 \beta$, and IL-6, by activating the nuclear factor- $\mathrm{\kappa} B$ and $\mathrm{p} 38$ mitogen-activated protein kinase signaling pathways $[8,9]$. IL-32 is also involved in several chronic inflammatory diseases, such as rheumatoid arthritis and COPD [10-12]. In addition to its role in

(c) The Author(s). 2018 Open Access This article is distributed under the terms of the Creative Commons Attribution 4.0 International License (http://creativecommons.org/licenses/by/4.0/), which permits unrestricted use, distribution, and 
inflammation, recent studies suggested that IL-32 is involved in liver fibrosis in patients with chronic hepatitis by affecting cytokine induction [13]. Although the precise effects of IL-32 on tissue fibrosis are largely unknown, IL-32 contains an RGD motif, which is known to bind several integrins [14]. Moreover, a 3-dimensional reconstruction model of IL-32 revealed that its structure was highly similar to that of the focal adhesion targeting (FAT) region of focal adhesion kinase (FAK). FAK-related non-kinase, a peptide with a structure similar to the FAT region, inhibits FAK signal transduction [15]. Because the integrin-FAK signaling axis is critical for the development of tissue fibrosis [16, 17], we predicted that IL-32 interrupts the signaling pathway by binding to these molecules, thereby inhibiting FAK activation and alleviating fibrosis.

We hypothesized that IL-32 $\gamma$ modulates fibrosis in chronic airway and lung diseases by disrupting the integrin-FAK signaling pathway. Here, we used murine models of chronic airway inflammation and bleomycin-induced pulmonary fibrosis to examine the role of IL-32 $\gamma$ in fibrosis of the airways and lungs, respectively. We also evaluated the role of $\mathrm{IL}-32 \gamma$ in mechanisms underlying fibroblast function.

\section{Methods}

Generation of murine models of airway inflammation and pulmonary fibrosis

To generate the bleomycin-induced pulmonary fibrosis model, mice were administered intratracheal injection of bleomycin (1 U/kg body weight) on day 2 . To evaluate the effect of IL-32 $\gamma$ treatment, mice were administered

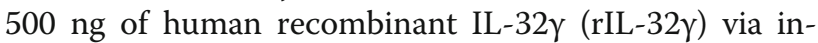
tranasal injection on days $1,2,14$, and 28 . In this model, rIL-32 $\gamma$ was injected intranasally; $1 \mathrm{~h}$ later, bleomycin was injected intratracheally. Mice were sacrificed at 30 days. To generate the chronic asthma model, wild-type (WT) mice were sensitized by intranasal administration of $22 \mu \mathrm{g}$ of ovalbumin (OVA) and $8 \mu \mathrm{g}$ of protease (Aspergillus melleus protease; Sigma, St. Louis, MO, USA) twice per week for 8 weeks, as previously described [23]. Mice were sacrificed at 58 days. To evaluate the effect of IL-32 $\gamma$ treatment, mice were treated with

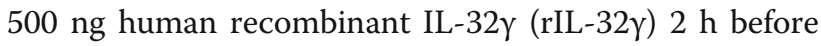
each immunization. IL-32 $\gamma$ transgenic (TG) mice on a C57BL/6 background were generated as previously described [18]. The Institutional Animal Care and Use Committee approved all experimental procedures (Animal Utilization Protocol 2014-14-013). Additional details are provided in the Additional file 1.

\section{Histopathologic examination and quantification of tissue fibrosis}

Lungs were removed and fixed in 10\% neutral-buffered formalin, embedded in paraffin, and sectioned $(4 \mu \mathrm{m})$.
Sections were subjected to Masson's trichrome staining and immunofluorescence staining using several antibodies. To quantify tissue fibrosis, we measured hydroxyproline levels in the tissue. Additionally, quantification graphs were drawn from intensity measurement data using the Image J program (NIH, Bethesda, MD, USA).

Additional details are provided in the Additional file 1.

\section{Cell culture and study design}

The human lung fibroblast cell line MRC-5 was purchased from the American Type Culture Collection (Manassas, VA, USA). Mouse embryonic fibroblasts (MEFs) obtained from IL-32 $\gamma$ TG mice were also used. MRC- 5 cells were seeded at $2 \times 10^{5}$ cells/well and stimulated with recombinant proteins. Expression of various cellular molecules was measured by Western blotting, reverse transcription-PCR, and semi-quantitative PCR. Additional details are provided in the Additional file 1. All in vitro experiments were conducted at least 3 times.

\section{Cell adhesion assay}

For crystal violet staining, 96-well culture dishes were coated with collagen (Advanced BioMatrix, Inc., San Diego, CA, USA) and seeded with MRC-5 cells. Plates were incubated for 30, 60, or $180 \mathrm{~min}$. Cells were washed with PBS to remove non-adherent cells, and adhered cells were stained with crystal violet. Additional details are provided in the Additional file 1.

\section{His pull-down assay and immunoprecipitation}

His-tagged IL-32 $\gamma$ was incubated with Ni-NTA agarose beads (Qiagen, Hilden, Germany), which were washed with buffer containing imidazole (Sigma). TGF- $\beta$-stimulated MRC-5 cells were lysed and centrifuged; the supernatant was incubated with Ni-NTA agarose-bound IL-32 $\gamma$. Proteins attached to the beads were subjected to immunoblotting. For immunoprecipitation, MRC-5 cells overexpressing flag-tagged IL-32 $\gamma$ were lysed and incubated with protein G Sepharose beads (GE Healthcare, Little Chalfont, UK) coated with an anti-flag antibody. Proteins were electrotransferred for immunoblotting. Additional details are provided in the Additional file 1.

\section{Live cell imaging of IL-32 $\gamma$}

MRC-5 cells were cultured in a $\mu$-Dish $35 \mathrm{~mm}$, High, IbiTreat (Ibidi GmbH, Martinsried, Germany) and treated with Flamma496-labeled IL-32 $\gamma$. After $10 \mathrm{~min}$, the cells were washed with medium. Fluorescence images were obtained under a Nikon Ti-E inverted I wamicroscope (Tokyo, Japan) equipped with PFS, iXon Ultra 897 EMCCD camera (Andor Technology, Belfast, UK), and excitation and emission filter wheels. 


\section{Statistical analysis}

All data are reported as the mean \pm standard error of mean. Means were compared using the Mann-Whitney test in GraphPad Prism software (version 4.0; GraphPad, Inc., La Jolla, CA, USA). A value of $p<0.05$ was considered statistically significant.

\section{Results}

\section{IL-32 $\gamma$ modulates fibrosis in mouse models of airway} inflammation and pulmonary fibrosis

First, histopathological analysis of bleomycin-induced lung fibrosis was conducted to determine the effect of IL-32 $\gamma$ on pulmonary fibrosis. Treatment with rIL-32 $\gamma$ significantly reduced collagen deposition and $\alpha$-smooth muscle actin (SMA) expression (Fig. 1a and b). Hydroxyproline levels showed a tendency to be lower in the bleomycin-induced fibrosis group treated with rIL-32 $\gamma$ than in the group without rIL-32 $\gamma$ treatment $(34.01 \pm$
7.24 vs. $25.52 \pm 3.66, p=0.048$; Fig. $1 c)$. Next, to determine the effect of IL-32 $\gamma$ on airway remodeling in chronic asthma, a murine model of chronic airway inflammation with subepithelial fibrosis was treated with rIL-32 $\gamma$. Treatment with rIL-32 $\gamma$ reduced peribronchial collagen deposition (Fig. 1d). This was accompanied by reduced expression of $\alpha$-SMA, a marker of activated fibroblasts, around the bronchi of treated mice (Fig. 1e). Figure 1f is a graph showing quantification of hydroxyproline. Hydroxyproline levels were significantly lower in the chronic asthma model treated with rIL-32 $\gamma$ (32.35 \pm 1.752 vs. $24.20 \pm 1.344, P=0.010$ ).

\section{rIL-32 $\gamma$ attenuates fibroblast activation}

Next, to determine whether IL-32 $\gamma$ affects fibrosis by regulating fibroblast activation, expression of fibronectin and $\alpha$-SMA was measured in the human fibroblast cell line MRC-5 after treatment with TGF- $\beta$ in the presence
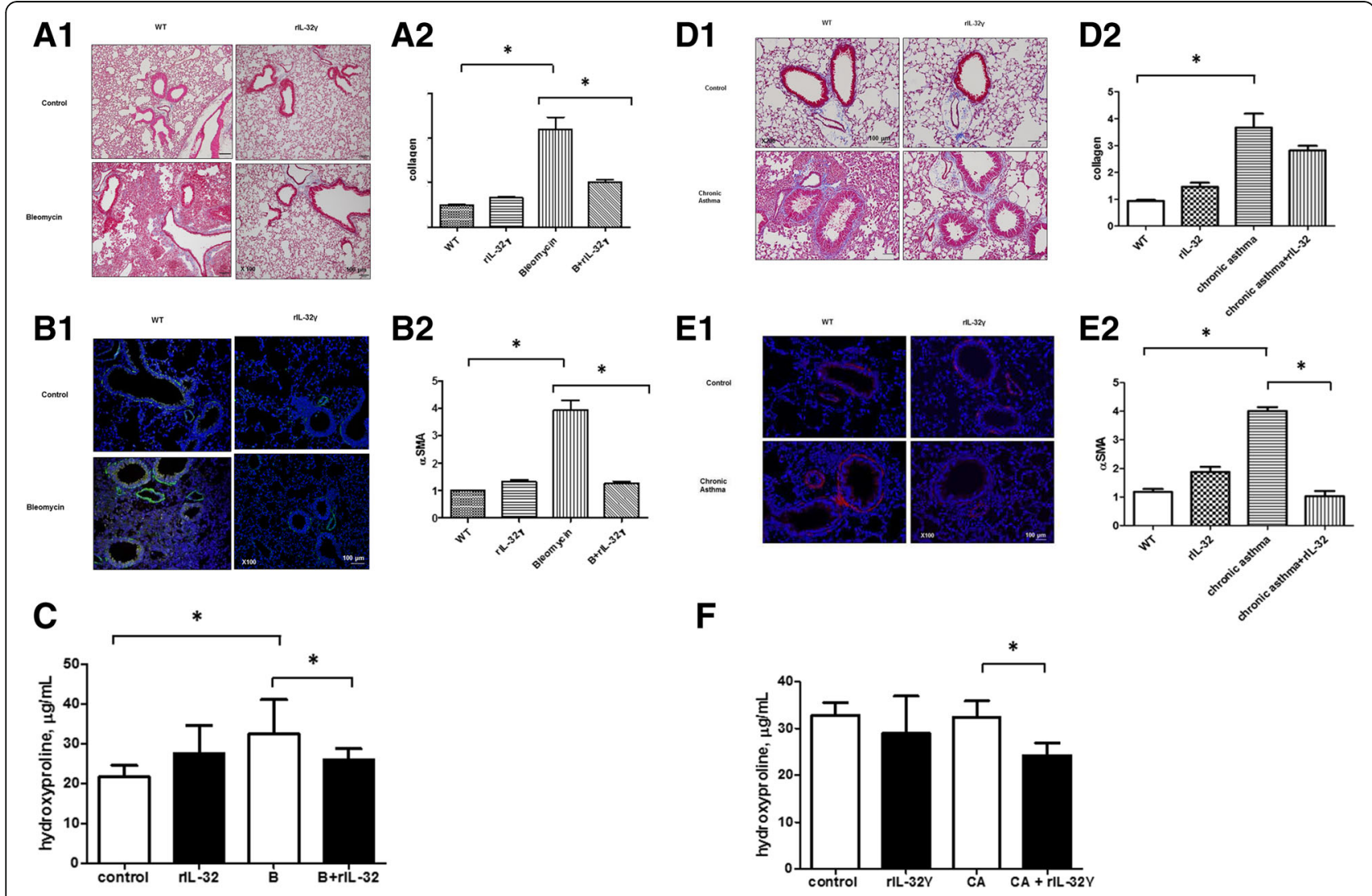

Fig. 1 Human IL-32y prevents fibrosis in chronic asthma and bleomycin-induced pulmonary fibrosis models. a Evaluation of collagen deposits in the lungs of bleomycin-induced mice using Masson's trichrome stain (original magnification: 100x). The quantification graphs of histological analysis in bleomycin-induced fibrosis groups. $\mathbf{b}$ Immunofluorescence analysis of a-SMA (green) expression in the lungs of bleomycin-induced mice. DAPI staining is blue (original magnification: 100x). c Hydroxyproline quantification. In the group with bleomycin-induced fibrosis treated

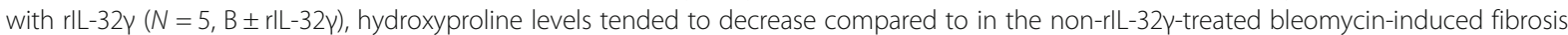
model $(N=6, B)(32.40 \pm 3.885$ vs. $26.70 \pm 1.287, P=0.166)$. $\mathbf{d}$ Evaluation of collagen deposition in the lungs of chronic asthmatic mice using Masson's trichrome stain (original magnification: $\times 200$ ). e Immunofluorescence analysis of a-SMA (red) expression in the lungs of mice with chronic asthma. DAPI staining is blue (original magnification: $\times 200$ ). $\mathbf{f}$ Hydroxyproline quantification graph. Similar results were obtained in each independent experiment, each using five mice per group ( $32.35 \pm 1.752$ vs. $24.20 \pm 1.344, P=0.010)$. ${ }^{*} P \leq 0.05$ 
or absence of rIL-32y. Fibronectin expression in rIL-32 $\gamma$-treated cells was significantly lower than that in untreated cells, whereas $\alpha$-SMA expression was slightly lower at early time points (Fig. 2a and b). However, overexpression of endogenous intracellular IL-32 $\gamma$ did not noticeably affect the production of fibronectin and

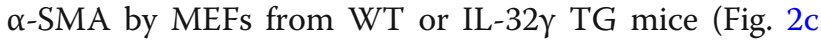
and Additional file 2: Figure S1). Endogenous IL-32 expression is shown in Fig. 2d.

Anti-fibrotic effect of rlL-32 $\gamma$ occurs independently of TNF-a Because IL-32 induces the production of TNF- $\alpha$ and vice versa, we next examined whether IL-32 $\gamma$ exerts anti-fibrotic effects by inducing TNF- $\alpha$ expression. First, we found that significant expression of IL-32 $\gamma$ mRNA was induced by TNF- $\alpha$, although no significant change in TNF- $\alpha$ mRNA expression was observed (see Additional file 3: Figure S2A and B). Similar to IL-32 $\gamma$, treatment with rTNF- $\alpha$ inhibited the expression of fibronectin and $\alpha$-SMA in TGF- $\beta$-stimulated MRC-5 cells
(Fig. 3a). However, IL-32 was not expressed by rTNF- $\alpha$ under IL-32 $\gamma$-knockdown conditions (Fig. 3b) and an anti-fibrotic effect of TNF- $\alpha$ was not observed in IL-32 $\gamma$-knockdown MRC-5 cells (Fig. 3c and Additional file 4: Figure S3A). Additionally, rIL-32 $\gamma$ inhibited fibronectin and $\alpha$-SMA expression after TNF- $\alpha$ inhibitor treatment (Fig. $3 \mathrm{~d}$ and Additional file 4: Figure S3B).

rIL-32 $\gamma$ does not appear to be involved in TGF- $\beta$ mediated Smad or non-Smad signaling

We next examined the effect of IL-32 $\gamma$ on activation of the Smad pathway, a well-known TGF- $\beta$-mediated signaling pathway. There were no significant differences in the expression of Smad signaling molecules (p-Smad 3, smuf2, and TGF- $\beta$ receptor 1 ), regardless of rIL-32 $\gamma$ treatment (Fig. 4a). Next, we examined whether the non-Smad pathway plays a role in the anti-fibrotic effects of rIL-32 $\gamma$. We found no significant differences in

A

A

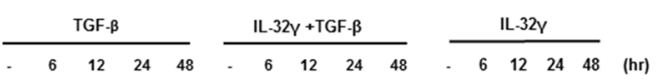
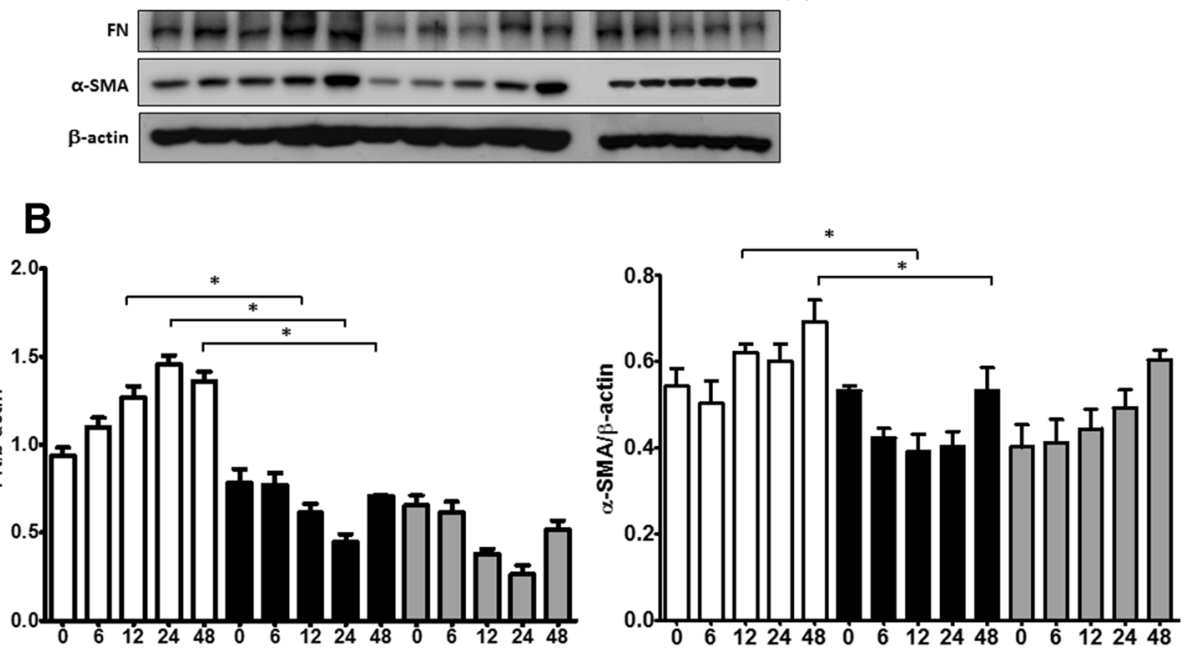

C

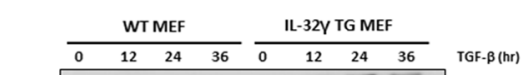

FN

$\alpha$-SMA

$\beta$-actin

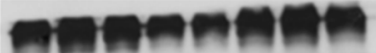

TGF- $\beta(\mathrm{hr})$

D

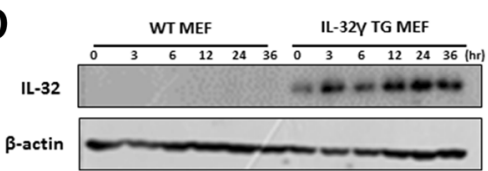

Fig. 2 Exogenous, but not endogenous, IL-32 $\gamma$ attenuates fibroblast activation. Fibronectin and a-SMA expression was detected in rIL-32 $\gamma$ (150 ng/mL)-pretreated MRC-5 cells after TGF- $\beta$ (5 ng/mL) stimulation. a. Quantification graphs is shown (b). Fibronectin and a-SMA expression are shown in IL-32y-expressing MEFs after TGF- $\beta(5 \mathrm{ng} / \mathrm{mL})$ stimulation $(\mathbf{c})$. Endogenous IL-32 expression (d). Data are representative of three independent experiments 


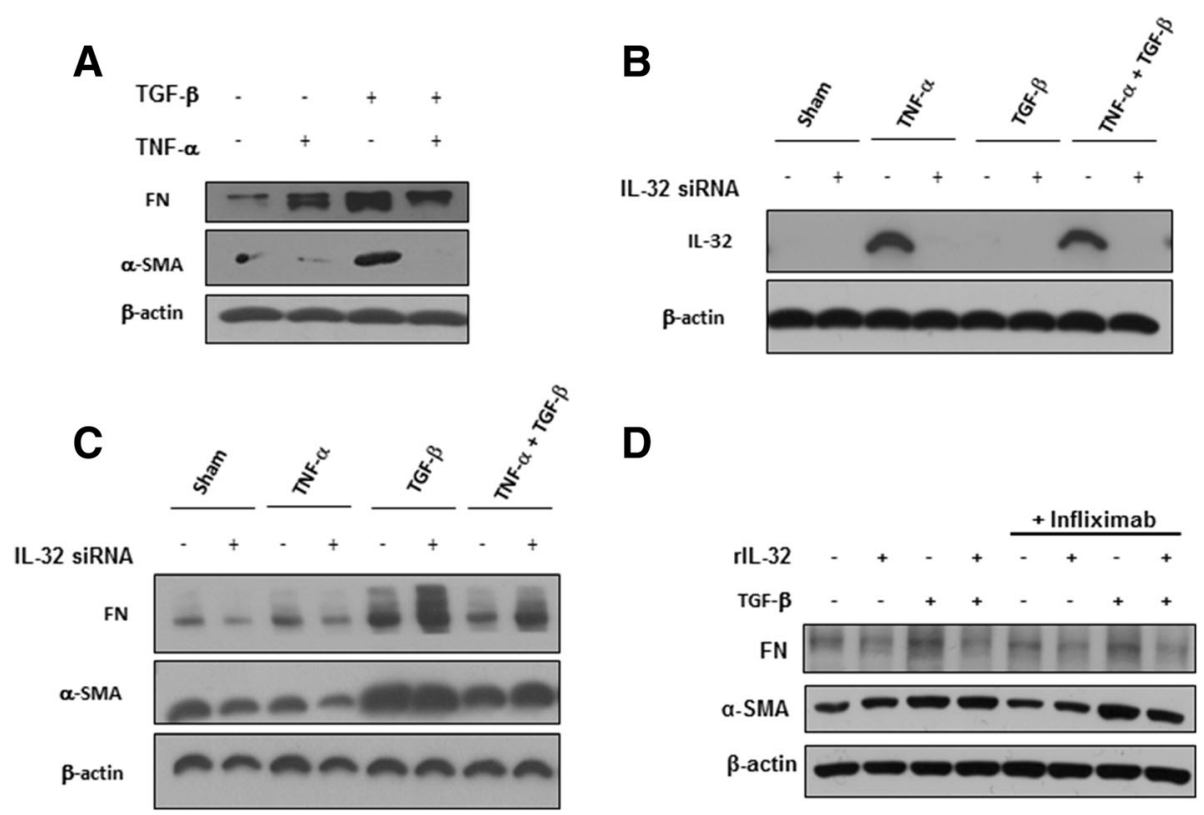

Fig. 3 Anti-fibrotic effects of rlL-32 $\gamma$ are independent of TNF- $a$. a Fibronectin and a-SMA expression in MRC-5 cells after $24 \mathrm{~h}$ of stimulation with TNF-a $(10 \mathrm{ng} / \mathrm{mL})$ and TGF- $\beta$ ( $5 \mathrm{ng} / \mathrm{mL})$. b MRC-5 cells were transfected with IL-32 siRNA and then stimulated with TNF-a (10 ng/mL) or TGF- $\beta$ $(5 \mathrm{ng} / \mathrm{mL})$. c Fibronectin and a-SMA expression in cell lysates was detected. $\mathbf{d}$ Infliximab-pretreated MRC-5 cells were stimulated with IL-32y $(150 \mathrm{ng} / \mathrm{mL})$ and TGF- $\beta(5 \mathrm{ng} / \mathrm{mL})$, and fibronectin and a-SMA expression in the cell lysate was detected. Results are representative of two independent experiments, each showing similar results

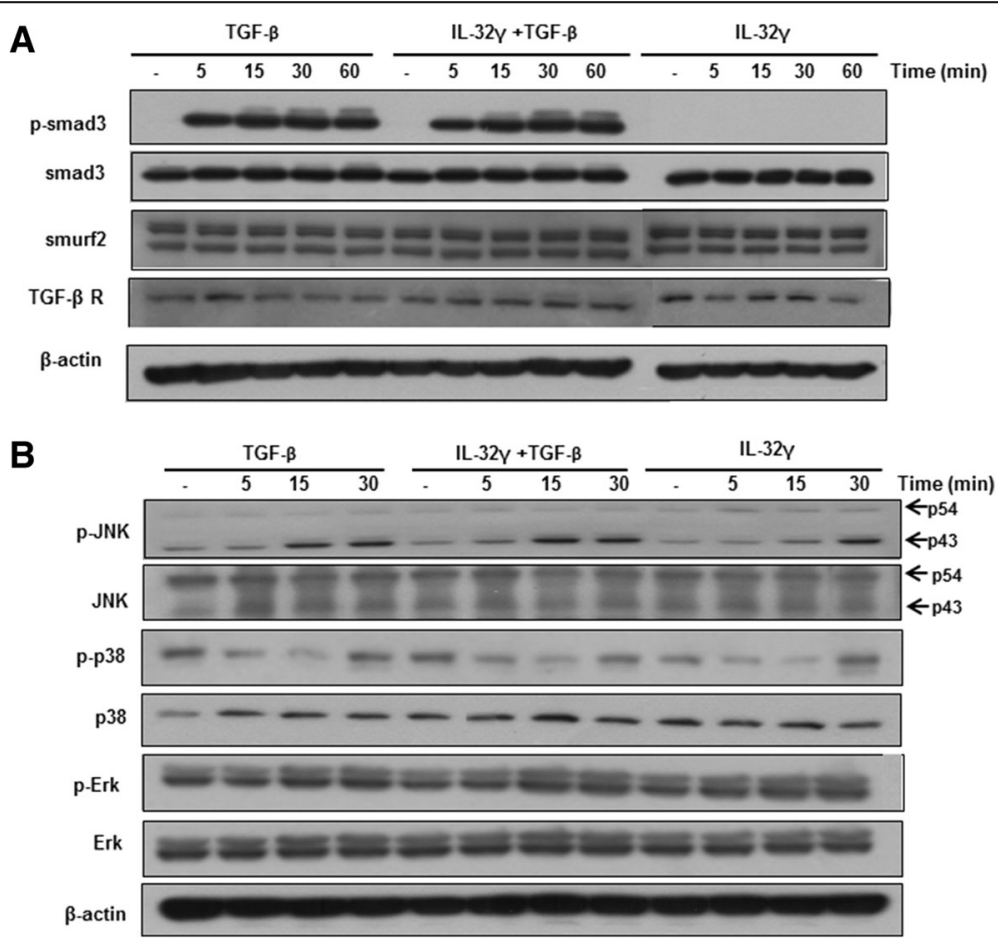

Fig. 4 rlL-32 $\gamma$ has no effect on TGF- $\beta$-mediated Smad or non-Smad signaling pathways. MRC-5 wells were stimulated with TGF- $\beta$ ( $5 \mathrm{ng} / \mathrm{mL}$ ) in the presence or absence of rIL-32 $\gamma$ and then harvested at the indicated times. Western blot analysis was performed to examine the expression of proteins in the Smad signaling (a) and non-Smad signaling pathways (b). Results are representative of three independent experiments 
JNK, Erk, and p38 activation between MRC cells treated with rIL-32 $\gamma$ and untreated cells (Fig. 4b).

\section{rIL-32 $\gamma$ inhibits integrin-mediated FAK/paxillin activation}

Next, we examined integrin-dependent activation of FAK and paxillin, a critical pathway in fibroblast activation, after treatment with the RGD tripeptide and integrin blocker. RGD peptide inhibited signaling by both FAK and paxillin in MRC- 5 cells stimulated with TGF- $\beta$ (Fig. $5 \mathrm{a}$ ). Interestingly, rIL-32 $\gamma$ inhibited FAK and paxillin signaling in a manner similar to that of RGD peptide (Fig. 5b).

To investigate how IL-32 $\gamma$ regulates the integrinFAK-paxillin signaling pathway, we performed a proteinprotein binding assay to determine whether IL-32 directly binds to integrin $\beta 3$, paxillin, or FAK. Both integrin $\beta 3$ and paxillin were detected in the total cell lysate and flow-through lanes, but no bands were detected in the wash and elution fractions (Fig. 5c). This suggests that these proteins do not directly bind to IL-32 $\gamma$. Additionally, an anti-flag-IL-32 $\gamma$ antibody did not immunoprecipitate with FAK (Fig. 5d).

\section{rlL-32 $\gamma$ is localized on the cell surface}

To determine the mechanism by which rIL-32 $\gamma$ inhibits activation of the FAK/paxillin pathway, we next examined the location of rIL-32 $\gamma$ by live cell imaging for 60 min. rIL-32 $\gamma$ was located outside of MRC-5 cells after
60 min, suggesting that it does not enter cells by endocytosis and is not degraded; therefore, IL-32 $\gamma$ acts extracellularly, at least during the period examined (Fig. 6, see Additional files 5 and 6: Video 1 and 2 in the online Supplement).

\section{rlL-32 $\gamma$ modulates the interaction between integrins and the extracellular matrix}

To examine the effect of IL-32y on integrin signaling, we examined the adhesion of MRC- 5 cells to collagen-coated plates in the presence/absence of rIL-32y. MRC- 5 cells adhered to collagen within $30 \mathrm{~min}$ in the absence of rIL-32 $\gamma$; however, the process was impeded in the presence of rIL-32 (Fig. 7a). Moreover, the number of spindle-shaped MRC-5 cells was much lower in the presence of rIL-32 $\gamma$, even after $30 \mathrm{~min}$ (Fig. 7b). Interestingly, rIL-32 $\gamma$ suppressed integrin/collagen-mediated activation of FAK and paxillin, which is typically induced by cell adhesion to collagen-coated plates in the absence of any other stimulation (Fig. 7c). Finally, we examined the effect of IL-32 $\gamma$ on integrin expression in MRC- 5 cells, as TGF- $\beta$ upregulates integrin expression. Semi-quantitative PCR revealed increased expression of integrin $\beta 3$ and reduced expression of integrin $\beta 8$ following TGF- $\beta$ stimulation. This pattern was not altered by IL-32 $\gamma$ treatment (see Additional file 7: Figure S4).
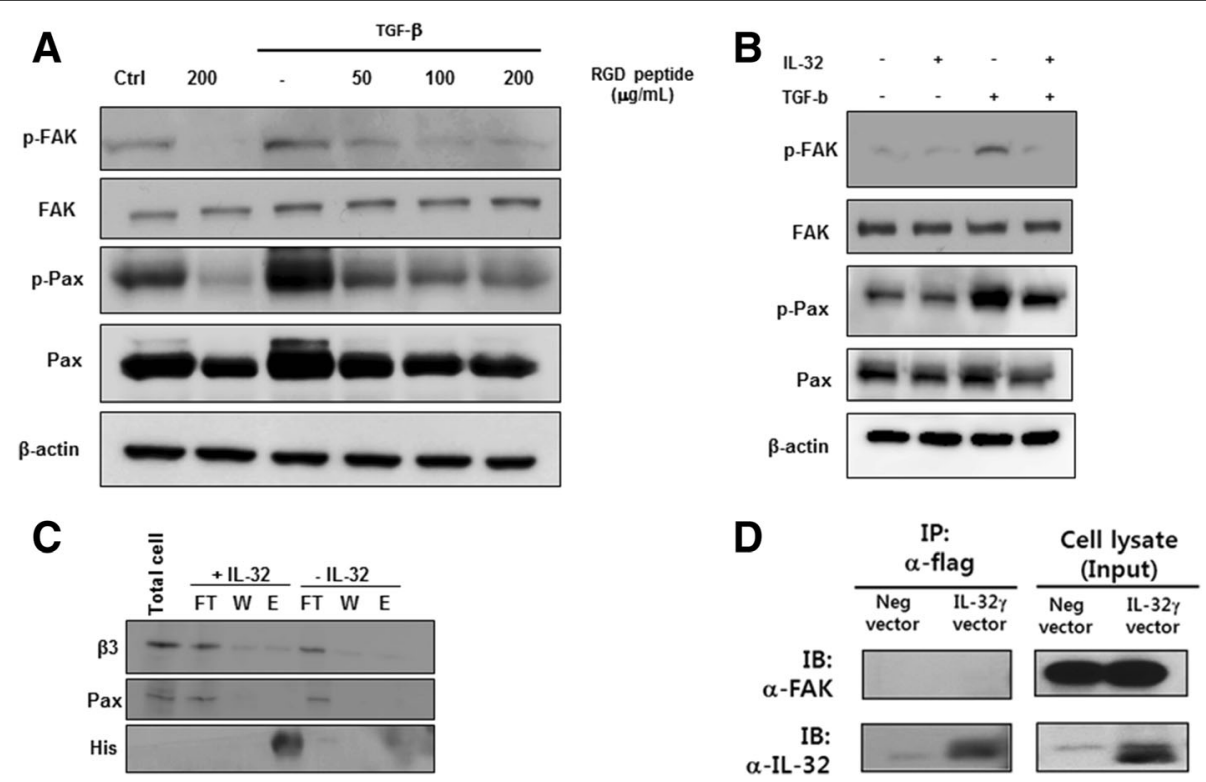

Fig. 5 rlL-32 $\gamma$ inhibits integrin-mediated activation of FAK/paxillin. Phosphorylation of FAK and paxillin was detected in TGF- $\beta$ ( $5 \mathrm{ng} / \mathrm{mL}$ )stimulated MRC-5 cells pretreated with an RGD peptide (a) or rlL-32y (b). Activated FAK and paxillin were detected after 24 h. Results are representative of three independent experiments. c MRC-5 cells were stimulated with TGF- $\beta$ for $24 \mathrm{~h}$, and His-tagged rlL-32 $\gamma$ was precipitated from cell lysates using Ni-NTA beads. Bound proteins were analyzed by Western blotting with antibodies specific for integrin $\beta 3$, paxillin, and the His-tag. $\mathbf{d}$ Flag-tagged IL-32 $\gamma$-overexpressing MRC-5 cells were stimulated with TGF- $\beta$ and harvested at $24 \mathrm{~h}$. Flag-tagged IL-32 $\gamma$ was then immunoprecipitated from cell lysates using an anti-flag antibody followed by immunoblotting with an anti-FAK antibody. Similar results were obtained from two independent experiments. FT, flow-through; W, wash; E, elution 


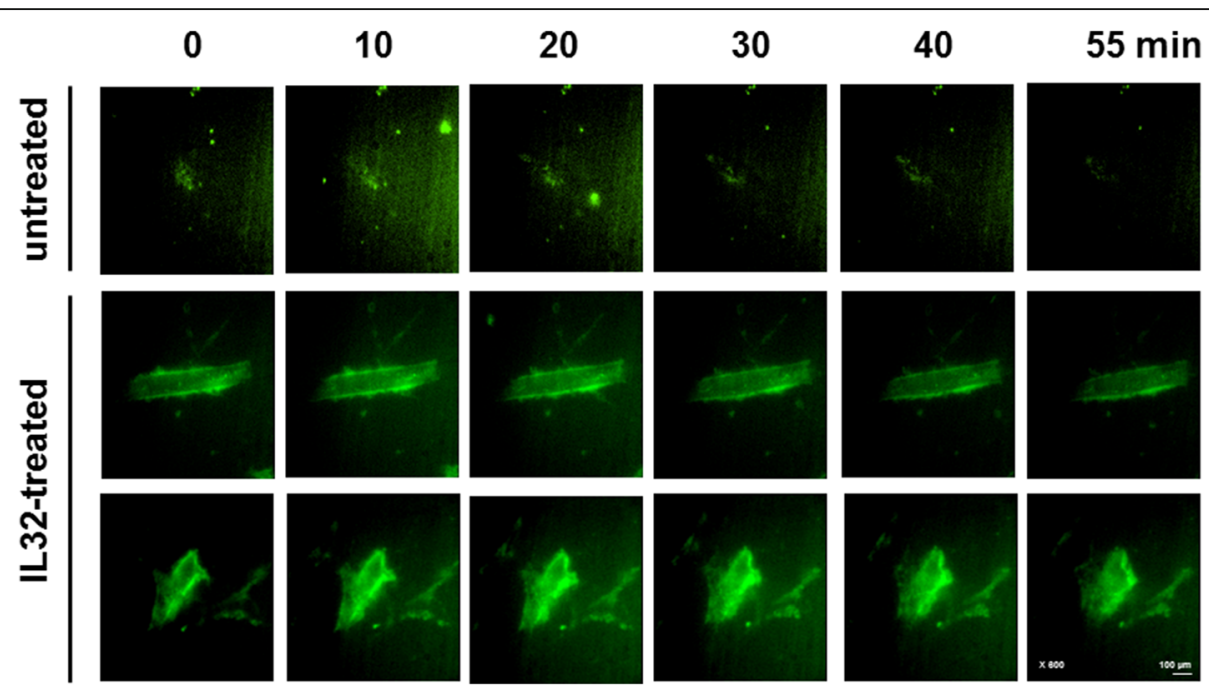

Fig. 6 rIL-32y localizes extracellularly. Live cell imaging of MRC-5 cells at 10-60 min post-incubation with Flamma496-labeled IL-32y (magnification, $600 x$; green color)
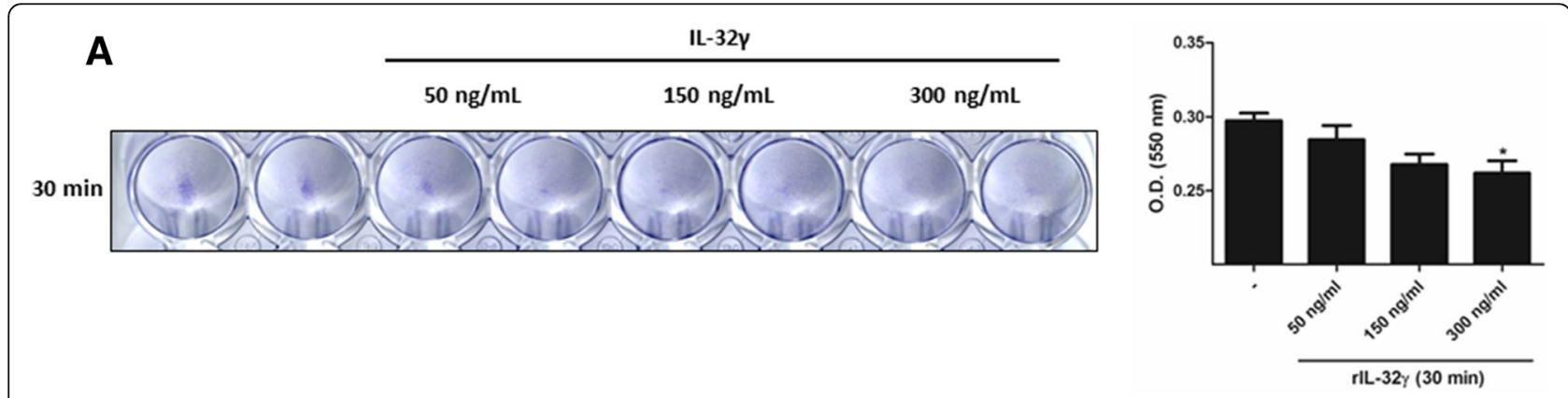

B
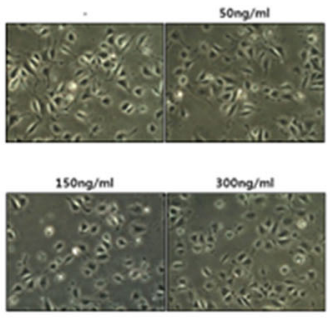

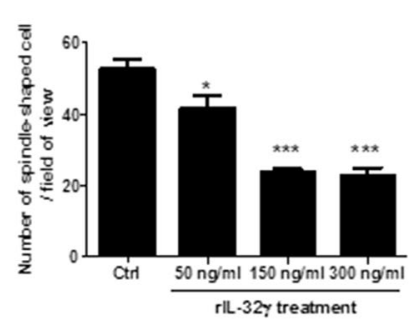

C

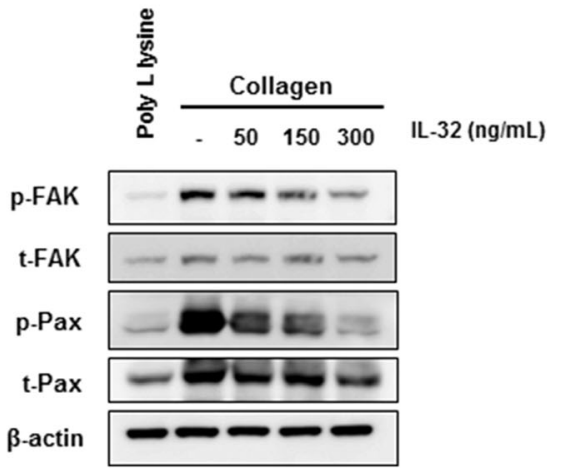

Fig. 7 rlL-32y modulates the interaction between integrins and the extracellular matrix. MRC-5 cells were plated on collagen-coated plates in the presence/absence of rIL-32y. a Adherent MRC-5 cells were stained with crystal violet immediately after the adhesion assay (left) and optical density values from the dissolved crystals are shown (right). Similar results were obtained from three independent experiments. ${ }^{*} P<0.05$ (b) Adherent cells were observed at 30 min under a microscope (original magnification: 100x). Similar results were obtained from two independent experiments. ${ }^{*} P<0.05,{ }^{* *} P<0.0001$ (c) Phosphorylation of FAK and paxillin was detected after MRC-5 cells attached to collagen-coated plates for $24 \mathrm{~h}$ in the presence/absence of rlL-32 $\gamma$. Similar results were obtained from two independent experiments 


\section{Discussion}

This study demonstrated the anti-fibrotic effect of IL-32 $\gamma$ both in vitro and in vivo. We showed that rIL-32 $\gamma$ regulates fibroblast activation by modulating the integrin-FAK signaling pathway. Thus, rIL-32 $\gamma$ may be useful for inhibiting tissue fibrosis in the clinical setting.

The mechanism of tissue fibrosis is closely related to that of wound repair, which is a normal healing process in injured tissues. However, dysregulated fibrosis can lead to severe organ dysfunction, which is typically irreversible and has a fatal outcome in many disease states. In the lungs, for example, progressive parenchymal fibrosis is a consequence of serious pulmonary fibrotic diseases such as idiopathic pulmonary fibrosis, leading to high mortality. Additionally, bronchial subepithelial fibrosis can cause irreversible fixed airway obstruction, as observed in chronic inflammatory airway diseases such as chronic severe asthma and COPD, which can become critical if untreated.

Although lung fibrogenesis is thought to result from chronic inflammation, numerous studies have suggested that fibrosis is not completely dependent on inflammatory processes and that anti-inflammatory therapeutic strategies are not always effective. Thus, therapeutic trials have shifted their focus from anti-inflammatory targets to anti-fibrotic targets, as many studies demonstrated that such mechanisms underlie the development of fibrosis [19-22]. However, therapeutic agents that effectively control fibrosis are lacking; therefore, there is an urgent need to identify novel molecules with potent anti-fibrotic activities.

IL-32, previously considered a pro-inflammatory cytokine, is a multifunctional protein with a potential role in lung diseases [12, 23-25]. We previously showed that IL-32 $\gamma$ modulates immune responses by recruiting IL-10-producing monocytic cells in a chronic asthma model [24]. Here, we observed that IL-32 $\gamma$ also exhibits a strong anti-fibrotic effect in a model of sub-bronchial fibrosis. Because chronic inflammation is a major factor driving the progression of fibrosis, its apparent suppressive effect on airway fibrosis may be completely dependent on the anti-inflammatory effects of IL-32 $\gamma$. Thus, we examined the modulatory effects of IL-32 $\gamma$ in a bleomycin-induced lung injury model, which is considered a prototype of tissue fibrosis but displays lower accumulation of immune cells in the lungs. This is of interest because IL- $32 \gamma$ is a putative immunomodulatory cytokine. The results of the current study suggest that IL-32 $\gamma$ has a novel function in lung fibrosis, as well as anti-inflammatory effects on chronic airway inflammation.

We also used human fibroblasts to further investigate the mechanism underlying the anti-fibrotic effect of IL-32 $\gamma$, as excessive accumulation of extracellular matrix produced by activated fibroblasts is a major pathological feature in tissue fibrosis, and any possible effects of inflammation in an animal can be excluded. MRC-5 cells were stimulated with TGF- $\beta$, which induces fibroblasts to differentiate into fibronectin- and $\alpha$-SMA-expressing myofibroblasts. We found that IL-32 $\gamma$ effectively inhibited expression of these activation markers upon TGF- $\beta$ stimulation. Previous studies showed that TNF- $\alpha$ and IL-32 $\gamma$ induce one another. Additionally, TNF- $\alpha$ inhibits the TGF- $\beta$-induced Smad signaling pathway [26-29]. Thus, we used cells in which IL-32 $\gamma$ had been silenced and a TNF- $\alpha$-blocking agent to determine the exact mechanism underlying the suppressive effect of IL-32 $\gamma$ on fibroblast activation. Furthermore, the intracellular pathways linked to the Smad and non-Smad signaling pathways were assessed. We found that the mechanism underlying the role of IL-32 $\gamma$ in fibrogenesis was not dependent on TNF- $\alpha$ expression, nor was it associated with activation of TGF- $\beta$ downstream of the Smad or non-Smad signaling pathways.

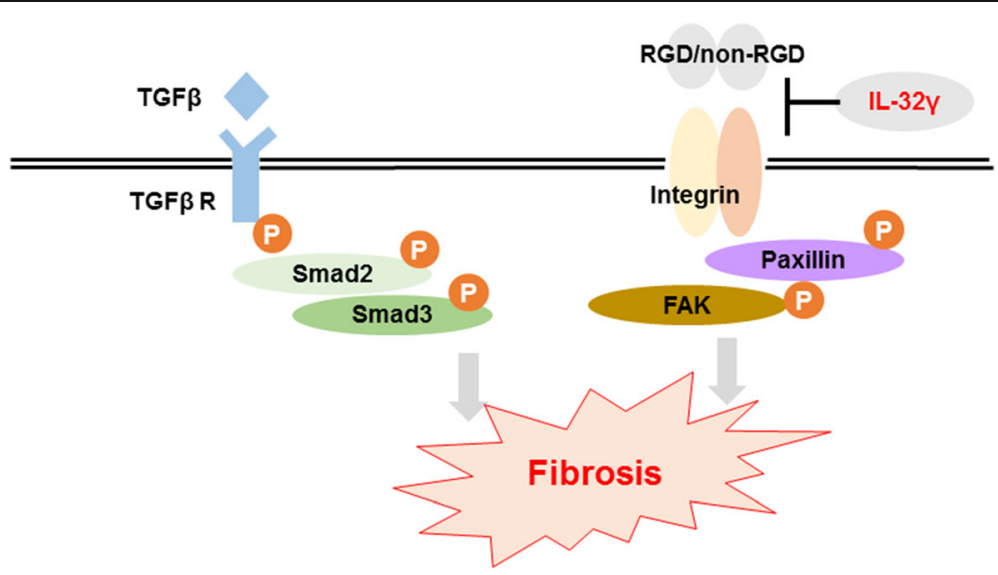

Fig. 8 Suggested role of IL-32y in the fibrosis pathway. Extracellular IL-32y suppresses activation of the integrin-FAK-paxillin signaling pathway to exert anti-fibrotic effects but has no effect on the TGF $\beta$-Smad signaling pathway 
Previous studies indicated that TGF- $\beta$-induced fibroblast activation depends on the integrin signaling pathway through FAK/paxillin activation [16, 30-32]. Protein structure modeling suggested that IL-32 $\gamma$ is involved in integrin activation and downstream signaling pathways [14, 33]. In fact, IL-32 $\gamma$ contains an RGD motif that binds to integrins; indeed, several isoforms of IL-32 bind to integrin $\alpha \mathrm{V} \beta 3$. In addition, IL-32 has a structure resembling the FAT region of FAK (similar to an FAK-inhibitory peptide). However, these studies examined only IL-32 $\alpha$ and $\beta$, although IL-32 $\gamma$ is considered the most active form [34].

We found that rIL-32y inhibited the phosphorylation of FAK and paxillin in TGF- $\beta$-stimulated fibroblasts without directly binding to these molecules. Based on these results, extracellular rIL-32 $\gamma$ regulates TGF- $\beta$-mediated fibroblast activation without entering the cell. Indeed, we observed that rIL-32 $\gamma$ treatment inhibited integrin-mediated cell adhesion, although rIL-32 $\gamma$ remained outside the cell. These results strongly suggest that IL-32 $\gamma$ is involved in the development of tissue fibrosis, likely by disrupting the binding between integrins expressed in the cellular membrane and the extracellular matrix.

No study has fully identified an IL-32-associated pathway in the context of fibrosis, raising the question of whether IL-32 is released by dead cells or via a specific secretory pathway. Notably, in the early phase of several diseases, IL-32 is produced by activated T cells, monocytes, and NK cells and acts as a pro-inflammatory cytokine that stimulates TNF- $\alpha$, IL- 6 , and IL- 8 production $[8,12,35,36]$. Because recent studies showed that IL-32 is not secreted [24, 37], IL-32 $\gamma$ released from injured epithelial cells in patients with chronic inflammatory diseases, including those with mycobacterium avium complex pulmonary disease and idiopathic inflammatory bowel disease [22, 37], may play a regulatory role in inflammation or tissue remodeling. For instance, our previous study showed that rIL-32 $\gamma$ suppresses chronic airway inflammation, which is closely associated with airway remodeling [24].

There were some limitations to the current study. First, to obtain more convincing and direct evidence to evaluate our hypothesis, mutations or deletions of the RGD motif of IL-32 $\gamma$ should be used. Second, our results do not clearly define the precise function of intracellular and extracellular IL-32y. Further studies are necessary to resolve these questions.

In summary, IL-32 $\gamma$ has anti-fibrotic effects likely by blocking the integrin-FAK-paxillin pathway (Fig. 8). Therefore, administration of rIL-32 $\gamma$ may play a pivotal role in modulating both inflammation and fibrosis in patients in which inflammation-related fibrosis pathways are activated.

\section{Conclusions}

The present study suggested that IL-32 $\gamma$ prevents tissue damage by regulating fibroblast activation in the chronic stage. The mechanism underlying this modulatory effect may involve disruption of integrin/FAK signaling cascades, without the need for IL-32 $\gamma$ to directly bind molecules involved in these cascades. Thus, IL-32 $\gamma$ is a new candidate for the treatment of lung fibrosis.

\section{Additional files}

Additional file 1: Additional methods detail. (DOC 46 kb)

Additional file 2: Figure S1. Extracelular IL-32 $\gamma$ suppresses fibroblast activation. Endogenous IL-32 $\gamma$ did not significantly suppress the expression of fibronectin and a-SMA. (TIF $202 \mathrm{~kb}$ )

Additional file 3: Figure S2. IL-32 $\gamma$ mRNA expression was induced by TNF-a. MRC-5 cells were stimulated with each cytokine including LPS $(1 \mu \mathrm{g} / \mathrm{mL})$, Poly I: C (10 $\mu \mathrm{g} / \mathrm{mL})$, TNF-a $(10 \mathrm{ng} / \mathrm{mL}), I L-32 \gamma(150 \mathrm{ng} / \mathrm{mL})$, TGF- $\beta$ ( $5 \mathrm{ng} / \mathrm{mL})$, and $\mathrm{IL}-1 \beta(10 \mathrm{ng} / \mathrm{mL})$. After $24-\mathrm{h}$ stimulation, mRNA level of IL-32 $\gamma(A)$ and TNF- $a(B)$ were measured by quantitative PCR. (TIF $71 \mathrm{~kb}$ )

Additional file 4: Figure S3. Anti-fibrotic effect of $\mathrm{rlL}-32 \gamma$ is independent of TNF-a. Anti-fibrotic effect of TNF-a was not observed in IL-32 $\gamma$ knockdown MRC-5 cells (A). rlL-32 $\gamma$ suppressed the expression of fibronectin and a-SMA after TNF-a inhibitor treatment (B). (TIF $96 \mathrm{~kb}$ )

Additional file 5: Supplementary video. (MP4 $2.42 \mathrm{mb}$ )

Additional file 6: Supplementary video. (MP4 $1.98 \mathrm{mb}$ )

Additional file 7: Figure S4. Integrin expression in activated fibroblast is not affected by rlL-32 $\gamma$. The integrin mRNA levels of $a 2, a v, \beta 1, \beta 3, \beta 5$, $\beta 8$, and the GAPDH mRNA level were determined by semi-quantitative PCR in MRC-5 after TGF- $\beta$ or rlL-32 $\gamma$ treatments. (TIF $111 \mathrm{~kb}$ )

\section{Abbreviations}

COPD: Chronic obstructive pulmonary disease; FAK: Focal adhesion kinase; FAT: Focal adhesion targeting; IL-32: Interleukin 32; MEFs: Mouse embryonic fibroblasts; OVA: Ovalbumin; rlL-32y: Recombinant interleukin 32 gamma; TG: Transgenic; TGF: Transforming growth factor; TNF: Tumor necrosis factor; WT: Wild-type; a-SMA: Alpha smooth muscle actin

\section{Acknowledgements}

We thank Dr. Joon Seo Lim from the Scientific Publications Team at Asan Medical Center for his editorial assistance in preparing this manuscript.

\section{Funding}

This work was supported by a National Research Foundation of Korea (NRF) grant, funded by the Korean government (NRF-2013R1A1A2064442).

\section{Availability of data and materials}

Source data and material will be available upon request.

\section{Authors' contributions}

GH Hong designed this study, acquired data, analyzed data, and drafted the manuscript. SY Park designed this study, analyzed data, drafted the manuscript, and revised the manuscript. HS Kwon, J Lee, SY Kim, CG Pack, S Kim, TB Kim, and HB Moon analyzed data and revised the manuscript. KA Moon acquired data and drafted the manuscript. YS Cho designed this study, analyzed data, drafted the manuscript, and revised the manuscript. All authors read and approved the final manuscript. All authors have accountability for all aspects of the work in ensuring that questions related to the accuracy or integrity of any part of the work are appropriately investigated and resolved. 


\section{Ethics approval and consent to participate}

The Institutional Animal Care and Use Committee of Asan Institute for Life Science approved all experimental procedures (animal Utilization Protocol 2014-14-013).

\section{Consent for publication}

Not applicable.

\section{Competing interests}

The authors declare that they have no competing interests.

\section{Publisher's Note}

Springer Nature remains neutral with regard to jurisdictional claims in published maps and institutional affiliations.

\section{Author details}

${ }^{1}$ Asan Institute for Life Science, Seoul, Korea. ${ }^{2}$ Department of Internal Medicine, Division of Allergy and Clinical Immunology, Asan Medical Center, University of Ulsan College of Medicine, 88 Olympic-ro 43-gil, Songpa-gu, Seoul 138-736, Korea. ${ }^{3}$ Department of Internal medicine, Division of Allergy and Respiratory Medicine, Konkuk University Medical Center, Seoul, Korea. ${ }^{4}$ Department of Internal Medicine, Jeju National University School of Medicine, Jeju, Korea. ${ }^{5}$ Department of Convergence Medicine, University of Ulsan, Seoul, Korea. 'Laboratory of Cytokine Immunology, Institute of Biomedical Science and Technology, College of Medicine, Konkuk University, Seoul, Korea.

Received: 22 March 2018 Accepted: 14 August 2018

Published online: 26 September 2018

\section{References}

1. Halwani R, Al-Muhsen S, Al-Jahdali H, Hamid Q. Role of transforming growth factor-beta in airway remodeling in asthma. Am J Respir Cell Mol Biol. 2011:44(2):127-33.

2. Murray LA. Commonalities between the pro-fibrotic mechanisms in COPD and IPF. Pulm Pharmacol Ther. 2012:25(4):276-80.

3. Wilson MS, Wynn TA. Pulmonary fibrosis: pathogenesis, etiology and regulation. Mucosal Immunol. 2009;2(2):103-21.

4. Chambers RC. Molecular targets in pulmonary fibrosis. CHEST. 2007:132(4):1311-21.

5. Kreuter M, Bonella F, Wijsenbeek M, Maher TM, Spagnolo P. Pharmacological Treatment of Idiopathic Pulmonary Fibrosis: Current Approaches, Unsolved Issues, and Future Perspectives. Biomed Res Int. 2015;2015:329481

6. Luzina IG, Todd NW, lacono AT, Atamas SP. Roles of T lymphocytes in pulmonary fibrosis. J Leukoc Biol. 2008;83(2):237-44.

7. Dahl CA, Schall RP, He HL, Cairns JS. Identification of a novel gene expressed in activated natural killer cells and T cells. J Immunol. 1992:148:597-603.

8. Kim SH, Han SY, Azam T, Yoon DY, Dinarello CA. Interleukin-32: a cytokine and inducer of TNFalpha. Immunity. 2005;22(1):131-42.

9. Netea MG, Azam T, Ferwerda G, Girardin SE, Walsh M, Park JS, Abraham E, Kim JM, Yoon DY, Dinarello CA, Kim SH. IL-32 synergizes with nucleotide oligomerization domain (NOD) 1 and NOD2 ligands for IL-1beta and IL-6 production through a caspase 1-dependent mechanism. Proc Natl Acad Sci U S A. 2005;102(45):16309-14.

10. Dinarello CA, Kim SH. IL-32, a novel cytokine with a possible role in disease. Ann Rheum Dis. 2006;65(Suppl 3):iii61-4.

11. Joosten LA, Netea MG, Kim SH, Yoon DY, Oppers-Walgreen B, Radstake TR, Barrera P, van de Loo FA, Dinarello CA, van den Berg WB. IL-32, a proinflammatory cytokine in rheumatoid arthritis. Proc Natl Acad Sci U S A. 2006;103:3298-303.

12. Calabrese F, Baraldo S, Bazzan E, Lunardi F, Rea F, Maestrelli P, Turato G, Lokar-Oliani K, Papi A, Zuin R, et al. IL-32, a novel proinflammatory cytokine in chronic obstructive pulmonary disease. Am J Respir Crit Care Med. 2008;178(9):894-901

13. Terasaki Y, Terasaki M, Urushiyama H, Nagasaka S, Takahashi M, Kunugi S, Ishikawa A, Wakamatsu K, Kuwahara N, Miyake K, Fukuda Y. Role of survivin in acute lung injury: epithelial cells of mice and humans. Lab Investig. 2013;93(10):1147-63.
14. Heinhuis B, Koenders MI, van den Berg WB, Netea MG, Dinarello CA, Joosten LA. Interleukin 32 (IL-32) contains a typical alpha-helix bundle structure that resembles focal adhesion targeting region of focal adhesion kinase-1. J Biol Chem. 2012;287(8):5733-43.

15. Nagoshi $Y$, Yamamoto G, Irie T, Tachikawa T. Expression of FAK-related nonkinase (FRNK) coincides with morphological change in the early stage of cell adhesion. Med Mol Morphol. 2006;39(3):154-60.

16. Thannickal VJLD, White ES, Cui Z, Larios JM, Chacon R, Horowitz JC, Day RM, Thomas PE. Myofibroblast differentiation by TGF- $\beta 1$ is dependent on cell adhesion and integrin signaling via focal adhesion kinase. J Biol Chem. 2003:278(14):12384-9.

17. Zhang L, Che C, Lin J, Liu K, Li DQ, Zhao G. TLR-mediated induction of proinflammatory cytokine IL-32 in corneal epithelium. Curr Eye Res. 2013;38(6):630-8.

18. Choi JBS, Hong J, Ryoo S, Jhun H, Hong K, Yoon D, Lee S, Her E, Choi W, Kim J, Azam T, Dinarello CA, Kim S. Paradoxical effects of constitutive human IL-32 $\gamma$ in transgenic mice during experimental colitis. Proc Natl Acad Sci U S A. 2010;107(49):21082-6.

19. Royce SG, Cheng V, Samuel CS, Tang ML. The regulation of fibrosis in airway remodeling in asthma. Mol Cell Endocrinol 2012, 351(2):167-175

20. Investigates KG. At the frontiers of lung fibrosis therapy. Nat Biotechnol. 2013:31(9):781-3.

21. Beckett PA, Howarth PH. Pharmacotherapy and airway remodelling in asthma. Thorax. 2003:58(2):163-74.

22. Bai X, Ovrutsky AR, Kartalija M, Chmura K, Kamali A, Honda JR, OberleyDeegan RE, Dinarello CA, Crapo JD, Chang LY, Chan ED. IL-32 expression in the airway epithelial cells of patients with Mycobacterium avium complex lung disease. Int Immunol. 2011;23(11):679-91.

23. Bang BR, Kwon HS, Kim SH, Yoon SY, Choi JD, Hong GH, Park S, Kim TB, Moon HB, Cho YS. IL-32y Suppresses Allergic Airway Inflammation in Mouse Models of Asthma. Am J Respir Cell Mol Biol. 2014;50(6):1021-30.

24. Meyer N, Christoph J, Makrinioti H, Indermitte P, Rhyner C, Soyka M, Eiwegger T, Chalubinski M, Wanke K, Fujita $H$, et al. Inhibition of angiogenesis by IL-32: possible role in asthma. J Allergy Clin Immunol. 2012;129(4):964-73. e967

25. Yamane $K$, Ihn $H$, Asano $Y$, Jinnin M, Tamaki K. Antagnistic effects of TNF-a on TGF-b signaling through downregulation on TGF-b receptor type II in human dermal fibroblast. J Immunol. 2003:171:3855-62.

26. Verrecchia F, Mauviel A. TGF-beta and TNF-alpha : antagonistic cytokines controlling type I collagen gene expression. Cell Signal. 2004;16(8):873-80.

27. Verrecchia F, Pessah M, Atfi A, Mauviel A. Tumor necrosis factor- inhibits transforming growth factor- /Smad signaling in human dermal fibroblasts via AP-1 activation. J Biol Chem. 2000:275(39):30226-31.

28. Franck Verrecchia $C T$, Erwin F, Wagner EF, Mauviel A. A central role for the JNK pathway in mediating the antagonistic activity of pro-inflammatory cytokines against transforming growth factor beta-driven SMAD3/4-specific gene expression. J Biol Chem. 2003;278(3):1585-93.

29. Mamuya FA, Duncan MK. aV integrins and TGF-beta-induced EMT: a circle of regulation. J Cell Mol Med. 2012;16(3):445-55.

30. Munger JS, Sheppard D. Cross talk among TGF-beta signaling pathways, integrins, and the extracellular matrix. Cold Spring Harb Perspect Biol. 2011;3(11):a005017.

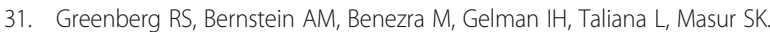
FAK-dependent regulation of myofibroblast differentiation. FASEB J. 2006;20(7):1006-8.

32. Joosten LA, Heinhuis B, Netea MG, Dinarello CA. Novel insights into the biology of interleukin-32. Cell Mol Life Sci. 2013;70(20):3883-92.

33. Choi JD, Bae SY, Hong JW, Azam T, Dinarello CA, Her E, Choi WS, Kim BK, Lee CK, Yoon DY, et al. Identification of the most active interleukin-32 isoform. Immunology. 2009:126(4):535-42.

34. Kim S. Interleukin-32 in inflammatory autoimmune diseases. Immune Netw. 2014:14(3):123-7.

35. Hong J, Bae S, Kang Y, Yoon D, Bai X, Chan ED, Azam T, Dinarello CA, Lee S, Her $E$, et al. Suppressing IL-32 in monocytes impairs the induction of the proinflammatory cytokines TNFalpha and IL-1beta. Cytokine. 2010;49(2):171-6.

36. Keswani A, Chustz RT, Suh L, Carter R, Peters AT, Tan BK, Chandra R, Kim SH, Azam T, Dinarello CA, et al. Differential expression of interleukin-32 in chronic rhinosinusitis with and without nasal polyps. Allergy. 2012;67(1):25-32.

37. Shioya M, Nishida A, Yagi Y, Ogawa A, Tsujikawa T, Kim-Mitsuyama S, Takayanagi A, Shimizu N, Fujiyama Y, Andoh A. Epithelial overexpression of interleukin-32alpha in inflammatory bowel disease. Clin Exp Immunol. 2007;149(3):480-6. 